\title{
EEG Signal Analysis Based Brain-Computer Interface
}

\author{
Hend Nooreldeen \\ Department of Industrial Electronics \\ and Control Engineering, Faculty of \\ Electronic Engineering, Menoufia \\ University, Egypt. \\ hend.abdelghaffar@el- \\ eng.menofia.edu.eg
}

\author{
Samir M. Badawy Departmen \\ of Industrial Electronics and Control \\ Engineering, Faculty of Electronic \\ Engineering, Menoufia University, \\ Egypt.. \\ drsamirb@gmail.com
}

\author{
Mohamed A. El-Brawany \\ Department of Industrial Electronics \\ and Control Engineering, Faculty of \\ Electronic Engineering, Menoufia \\ University, Egypt. \\ melbrawany@el-eng.menofia.edu.eg
}

\begin{abstract}
Brain Computer Interface (BCI) systems translate raw acquired brain signals into commands to control an external device. Advancement in bio- medical signal processing techniques has directed the Electroencephalography (EEG) signals not only as a diagnostic tool for brain disease, but also as a controller in Brain Computer Interface field. In this paper, we present an EEG data analysis to study how EEG activity changes with right and left imagery hand movements as a step toward controlling machines by thoughts. In this paper, features from analysis of power spectrum, event related potential and time frequency for EEG showed that right hand imagery decreases the activity of hand area in the brain left side and left hand imagery decreases the activity of hand area in the brain right side. The results successfully show that motor imagery EEG phenomena can be utilized in a BCI based motor restoration.
\end{abstract}

Keywords- Brain-computer interface (BCI), Electroencephalography (EEG) signals, motor imagery EEG(MI-EEG).

\section{INTRODUCTION}

EEG signals are measure for brain neural activity, which change according to task performed by a person [1]. The EEG has several medical applications. For example, EEG has been used as a brain diseases diagnostic method [2] Also, neuroscience advances, brain imaging technologies and computing have provided us with the opportunity to directly interface the brain with a computer, thereby creating a control and communication system that bypasses peripheral muscles and nerves to enable interaction through brain activity alone, this is known as brain-computer interface[3][4][5] . BCI depends on voluntarily modifying user mental state to control a device whereas a pattern analysis method concurrently tries to determine the parallel change of EEG signals[6]. fig. 1shows the common structure for BCI system. acquired brain signals at electrodes located on the scalp or in the head are processed to obtain determined signal features .These features are converted to control signals to control machines. EEG methods commonly executed for BCIs depend on activity of sensorimotor rhythms (SMR) measured during movement imagery and permit establishing sensorimotor rhythm-based BCI (SMR BCI)or motor-imagery BCI (MI BCI) [7]. experimentally MI-BCI can control devices like wheelchairs[8]. Wolpaw and McFarland [9] showed that depending on imagination of left and right hand movement a cursor two-dimensional control was possible. The work was increased to a cursor three-dimensional control depending on imagination of foot, left, and right hand movement[10]. The introduction of four-class BCIs enabled users to fly a virtual helicopter[11] and a robotic quad-copter in three dimensions [12]. imagining moving and relax the two hands made the helicopter fly forward and reverse and imagining moving left and right hand made the helicopter turn around left and right. The work in [11] was extended in [13] to a six-class BCI. feet and tongue movement imagery made the exceed dimensional control. Previous mentioned studies depend on more than two electrodes . As shown in [7], handedness has an influence on sensorimotor rhythm (SMR) distribution and BCI function. During imagery of left-hand movements, left-handers have lower precision and poorer SMR suppression in the alpha band $(8-13 \mathrm{~Hz})$. similar results have been observed in our work where the difference is clearer in case of right than left hand imagery. In [14] quantitative EEG changes were extracted from various combinations of channels due to movement imagination. To determine frequency bands specific to the subject, a characteristic filter bank popular spatial algorithm was introduced in [15] to show how EEG activity changes with left and right hand imagery movements depending on two channels and this require further computation.

In this paper, we show a study of how EEG activity changes with imagery of left and right hand movements depending on only two electrodes as a step toward developing BCI based assistive devices and toward portable BCI. The analysis techniques implemented in our work include power spectrum, event related potentials and the EEG signal time frequency analysis. Those analysis techniques successfully facilitate classification between imagination of left and right hand movements in a multidimension way depending on two electrodes. The result showed that we can classify right and left hand imagery movements in either frequency, time, or combined timefrequency domain.

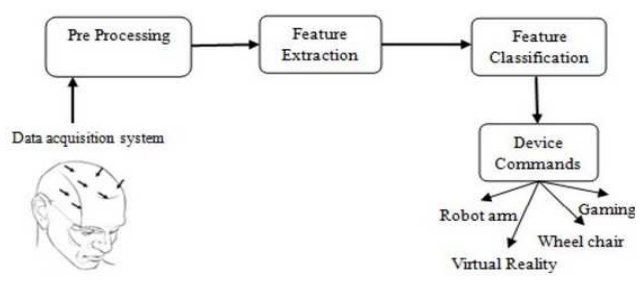

Fig.1. Architecture of an EEG-based BCI. 
Remainder of paper is arranged in the following manner: Section II explains methods and materials used. Section III introduces results and its discussion. The conclusions and future work are presented in section IV.

\section{MATERIALS AND METHODS}

In this study, we used dataset IIb of the BCI competition IV[16]. It is made up of EEG data of 9 normal persons. All users were sitting in an armchair placed 1 meter far at eye level, watching at screen monitor. Five sessions for each subject are introduced, three sessions with feedback and two sessions without feedback (screening). At a sample rate of $250 \mathrm{~Hz}$, bipolar recordings on $\mathrm{Cz}, \mathrm{C} 4$, and $\mathrm{C} 3$ were measured [17]. It was filtered between $(0.5-100) \mathrm{Hz}$, and a notch filter at $50 \mathrm{~Hz}$ was enabled. Two classes were found in the paradigm, namely imagination of left (class 1) and right (class2) hand movement. EEG data from 120 repetitions of each MI class per person is included in the session without feedback. EEGLAB is a free MATLAB toolbox for analyzing electrophysiological data [18]. Users may use EEGLAB to call a variety of data formats, preprocess data (filter, re-sample, epoch, average), visualize data (eventbased potentials, signal browser, power spectra), and execute independent component analysis (ICA), implement many time/frequency analysis techniques like event related spectral perturbation (ERSP) and inter-trial coherence (ITC). The extensible plug-in architecture enables contribution of new capabilities like connectivity estimation, source localization or creation online braincomputer interfaces[19].This work uses EEGLAB to analyzes the EEG rhythms reactivity caused by imagination of hand movements . Also removing EEG ocular artifacts was made by the AAR plug-in., it is a group of scripts that execute many important methods to automatically correct muscular and ocular artifacts in EEG signal[20]. Results can be used as an input to EEG classifier which can be used in a BCI for motor restoration.

\section{A. Preprocessing steps}

The first step of preprocessing is bringing the EEG data files into the EEGLAB and determining the sampling frequency of the data. The location of channels was imported to obtain information about the recording electrodes necessary for plotting EEG scalp maps or calculating data part source locations[21]. EEG signal has a quite poor resolution in space because of cconduction of volume through brain layers. To improve the spatial resolution, spatial filters are used. One of the important common spatial filtering techniques is bipolar recording[22][23]. It measures the difference in potential between two "active" electrodes hence improving the signal to noise ratio by lowering the common noise of the two electrodes. Dataset IIb from BCI competition IV contains three bipolar channels data. Eliminating noises in EEG can be done in the pre-process stage in time domain, thereby improving signal quality without losing relevant information by enhancing the ratio of signal to noise and improving performance of EEG analysis. During recording the EEG, the signal is contaminated by various artifacts as an MA (Muscle artifact), EBA (Eye blink artifact).In this study, the AAR (automatic Artifact Removal) plug-in is used to get rid of signal artifacts. It is a collection of scripts that execute many important strategies for correcting ocular and muscular artifacts automatically. The toolbox is carried out as a plug-in in EEGLAB. Many fully automatic strategies for correcting ocular (EOG) artifacts, and one automatic strategy for correcting muscle (EMG) artifacts are included in the existing versions[20]. In this dataset, the EEG signal is affected by ocular (EOG) artifacts. The AAR plug-in has been used to remove EOG artifacts[20] . EOG artifact was removed using Conventional Recursive Least Squares (CRLS) method (filter order(M): 3, forgetting factor (lambda): 0.9999, initial state of the filter (sigma): 0.01)[24]. Finally, between (8:30) Hz the EEG signal is bandpass filtered. The data epochs according to necessary events were derived from the filtered data, followed by the elimination of irrelevant epoch baseline offsets, in order to research the event based EEG dynamics of continuously registered data. Cue onset left (class 1) and Cue onset right (class 2) epochs were derived from two cases.

\section{B. Analysis of EEG Signals}

Movement or the planning for movement is usually followed by a decrease in specific sub bands such as mu $(\mu)$ and beta $(\beta)$ frequency bands and activity-over, specifically contralateral to the movement causing EventRelated Desynchronization (ERD) [25][26][27]. Rhythm rise, also known as "Event-Related Synchronization" (ERS), happens after activity and throughout resting. The $\mu$ rhythms are made up of a variety of $(8-13) \mathrm{Hz}$ rhythms that vary in frequency, position, and relationship to motor output or sensory input. Hand area $\mu$ rhythm is the prominent $\mu$ rhythm found on the scalp nearby electrodes c3 and c4 [22][23][25][27]. MI EEG-based BCI is considered active $\mathrm{BCI}$ as it does not require additional stimulation devices and thus the user can interact with the environment more freely. ERD and ERS can occur independently of activity in the brain's usual peripheral nerve and muscle output channels, and can thus be used as the foundation for an individual BCI. The ERS/ERD can be measured in both time and space and shown as time courses or charts[28] [26].

\section{Power Spectrum Analysis}

The time domain signals obtained from EEG amplifier reflect only one side of EEG. For precise analysis, we looked at the signal in the frequency domain. Spectral plot shows scalp maps of power at specified frequencies and plot spectra of specified data channels or components. As a result, it's simple to figure out which parts of the brain are active during the event[29].

\section{Event Related Potential (ERP) plots}

Scalp-recorded ERPs are voltage changes in the ongoing EEG. The study of 1-dimensional ERP averages has dominated the field of electrophysiological data analysis, because it leads to a reduction in event-unrelated EEG activity so increases the signal / noise ratio-relative to artifacts [27] [21] .

In this paper, channel ERP image plots have been used. The ERP plot displays a series of data epochs trial by trial. Each horizontal line in a colored rectangular ERP picture reflects a possible series of time throughout an experimental trial. Rather than single trials activity plotting as left-to-right traces with potential encoded by a data trace's ordinate, trials are depicted as horizontal lines with varying color values that reflect the trial's potential at every time point [29]. 


\section{E. Time frequency Analysis}

The EEG signal is dynamic by nature since it changes constantly in time domain and its energy distribution are scattered, thus the best characterized information that represent the signal of EEG can be obtained from the joint time-frequency domain. The EEGLAB toolbox has been used to provide two time/frequency analysis measures:

a) Channel time-frequency: which returns time locked estimates and plots of event related spectral perturbation (ERSP) and inter trail coherence (ITC) changes for a series of input events in a single data channel.

b) Channel crossing-coherence: return estimates and plots of event related spectral coherence [22] [18].

\section{RESULTS AND DISCUSSION}

\section{A. Removing EOG Artifact and data filtering.}

fig. 2 shows the EEG signal contaminated with EOG artifact while the result of algorithm of automatic EOG correction is shown in fig. 3. The blinks were almost completely gone, while the EEG remained unaffected. fig. 4 shows the filtered EEG signal from (8:30) Hz.

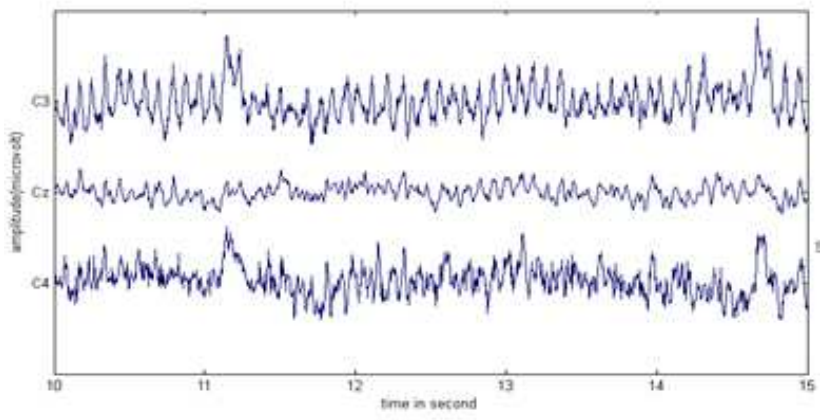

Fig. 2. original EEG signal with EOG artifact.

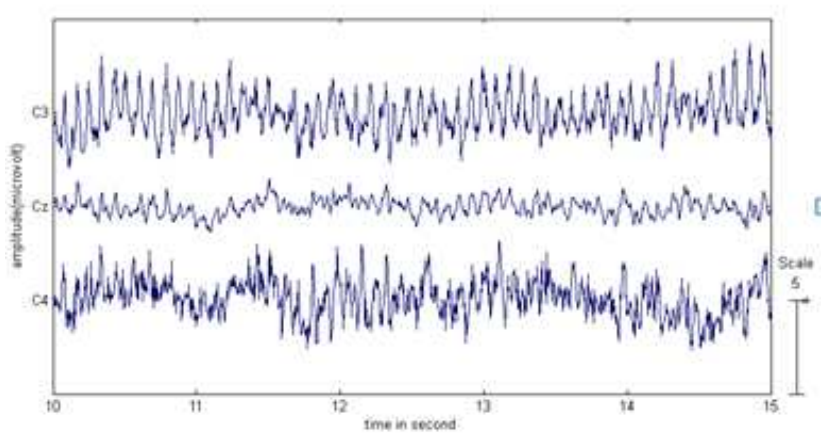

Fig. 3. EEG with EOG removed.

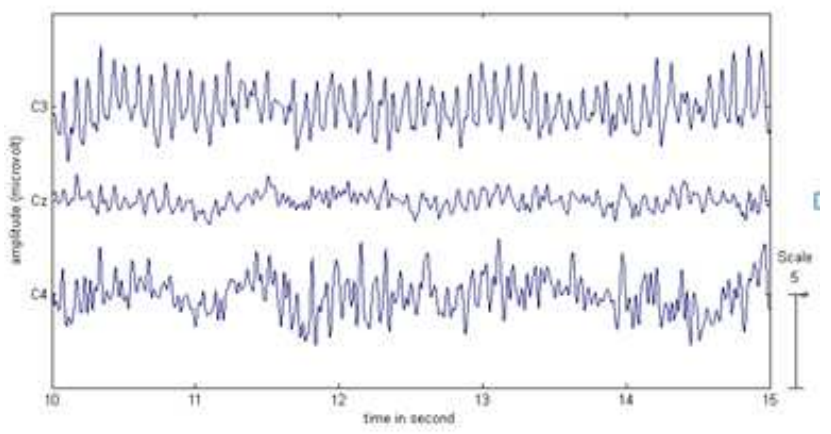

Fig. 4. Filtered EEG.

B. Power Spectrum Analysis. fig. 5 and fig. 6 show the logarithmic power spectral density of left and right motor imagery, respectively. It is evident from the figures, in the right hemisphere, there is a substantial drop in mean power during left motor imagery (the sensorimotor region on the right brain side monitors left-hand movement). Also, in the left hemisphere, there is a substantial drop in mean power during right motor imagery (the sensorimotor region at the brain left side that monitors right- hand movement).

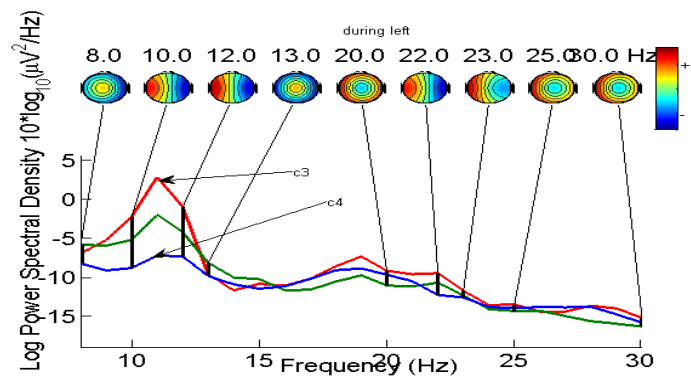

Fig. 5. Scalp distribution of power during left hand imagery.

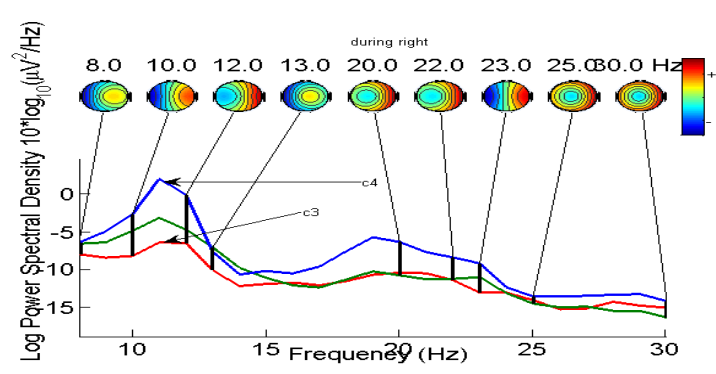

Fig. 6. Scalp distribution of power during right hand imagery.

\section{Channel ERP Image plots.}

The left hand imagery's channel ERP picture shows a lowering in the event based potentials of the sensorimotor region right side and a raise in event based potentials on the left side as shown in fig. 7. As shown in fig. 8, the right hand imagery's channel ERP picture shows a lowering in the event based potentials on the sensorimotor area left side and a raise in event related potentials on the right side, this mean that the sensorimotor rhythms are desynchronized during imagination of hand movements.
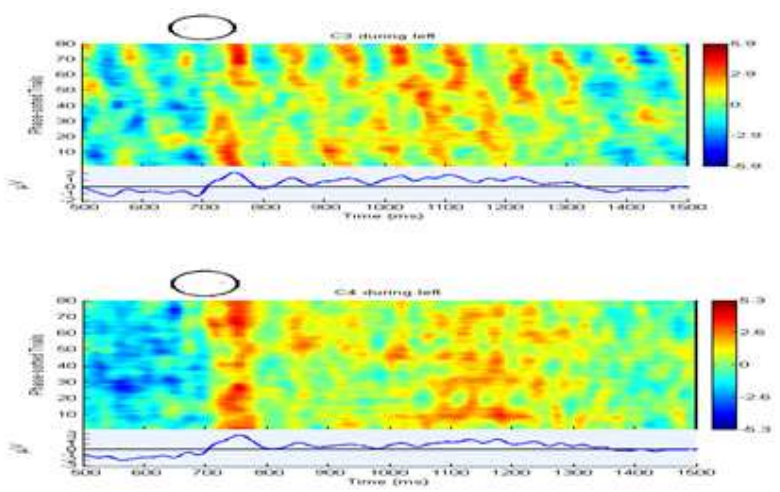

Fig. 7. channel ERPs at $\mathrm{C} 3$ and $\mathrm{C} 4$ electrode positions during imagery of left hand. 

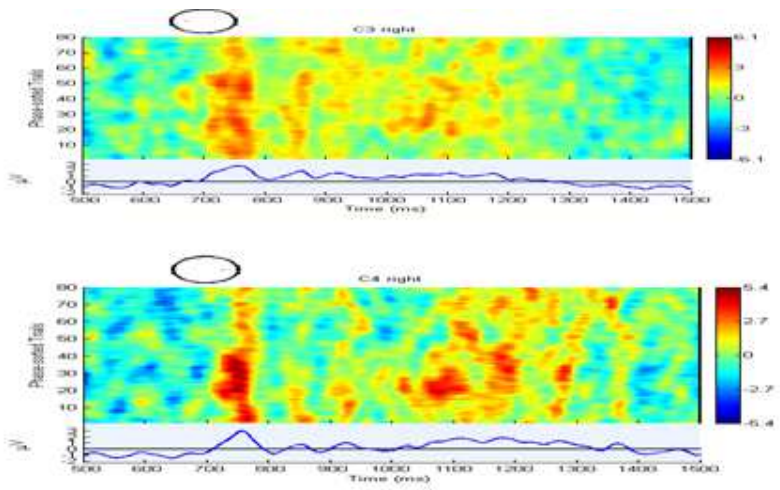

Fig. 8. channel ERPs at $\mathrm{C} 3$ and $\mathrm{C} 4$ electrode positions during right hand imagery.

D. Comparison of channel ERPS

The contrast of the channel c3, c4 ERPs enhances the conclusion drawn from the ERP picture as shown in fig. 9 .
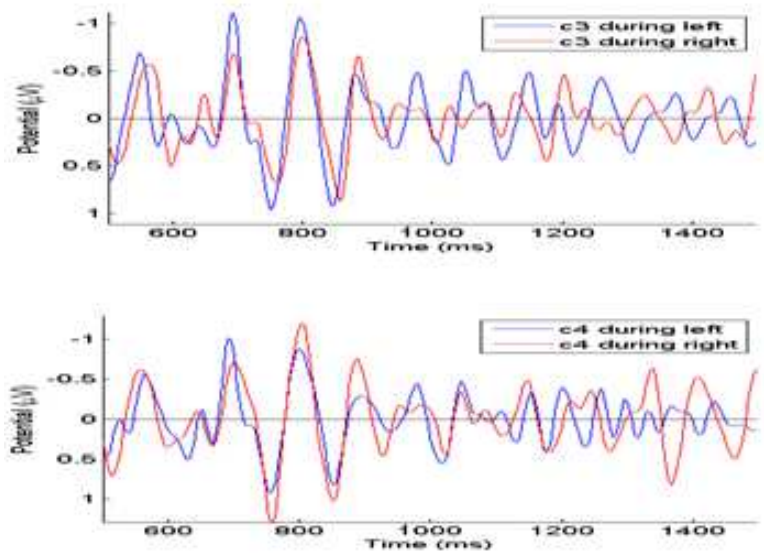

Fig. 9. ERP Comparison of left and right imagery at $\mathrm{C} 3$ and $\mathrm{C} 4$ electrodes positions.

E. Time frequency Plots

ERSP plot of C3 electrode of imagery of right hand demonstrates a lowering in the spectral power in the $\mu$ and $\beta$ rhythm around $8-30 \mathrm{~Hz}$, For hand motor imagery, this indicates $\mu$ rhythms desynchronization in the corresponding hand regions as shown in fig. 10. Moreover, the C4 electrode ERSP plot of imagery of left hand illustrate a lowering in the spectral power around $8-30 \mathrm{~Hz}$, which shows $\mu$ and $\beta$ rhythms desynchronization in the corresponding hand regions of imagination of hand movement as shown in fig. 11 .

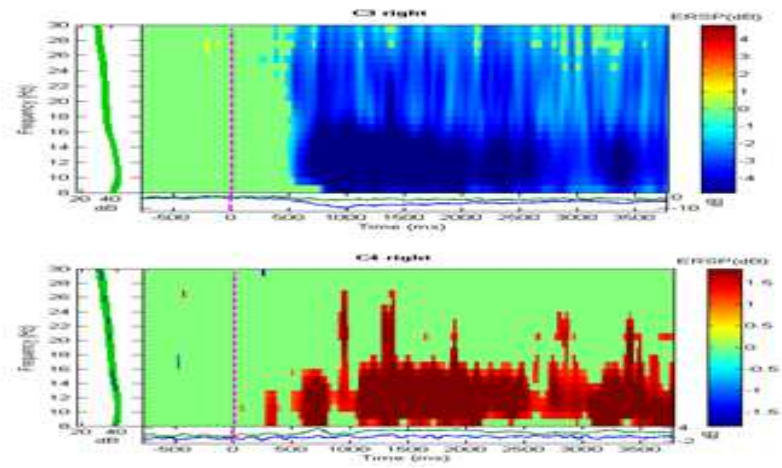

Fig. 10. ERSP plot at $\mathrm{C} 3$ and $\mathrm{C} 4$ electrode positions during imagery of right hand.
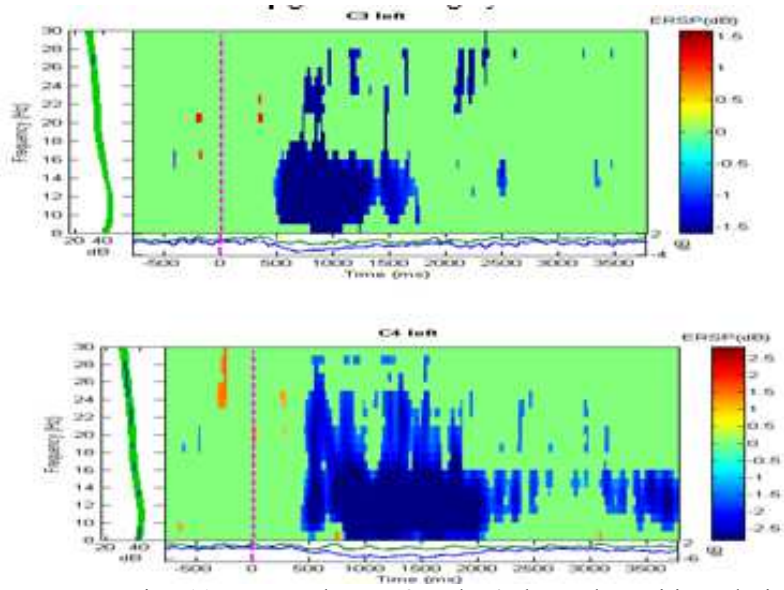

Fig. 11. ERSP plot at $\mathrm{C} 3$ and $\mathrm{C} 4$ electrode positions during imagery of left hand.

\section{CONCLUSION AND FUTURE WORK}

Results of this paper prove that imagination of right and left hand movement may alter activity of neural in primary sensorimotor regions, resulting in the changes of the $\mu$ and $\beta$ rhythms. Moreover, the results show that the distinction between electrode c4and c3 is more clear in case of right hand imagery. The results of this work can be used as a base in the classification stage for BCI and device control. Work in the future will use the results of this study to directly extract quantitative features to characterize between right and left -hand imagery and monitor embedded rehabilitation robot to assist patients with extreme paralysis in controlling their environmental and communicating with the outside world.

\section{REFERENCES}

[1] M. K. Ahirwal and N. D. Londhe, "Power spectrum analysis of EEG signals for estimating visual attention," Int. J. Comput. Appl. (0975 8887), vol. 42, no. 15, pp. 22-25, 2012, doi: 10.5120/5769-7993.

[2] X. Gu et al., "EEG-based brain-computer interfaces (BCIs): a survey of recent studies on signal sensing technologies and computational intelligence approaches and their applications," arXiv:2001.11337v1 [eess.SP], pp. 1-22, 2020.

[3] J. R.Wolpaw et al., "Brain - computer interface technology: a review of the first international meeting," IEEE Trans. Rehabil. Eng., vol. 8, no. 2, pp. 164-173, 2000, doi: 10.1109/TRE.2000.847807.

A. Roman-Gonzalez, "EEG signal processing for BCI applications," Adv. Intell. Soft Comput., vol. 98, pp. 571-591, 2012, doi: 10.1007/978-3-64223187-2_36.

H. S. Kisakye, "Brain computer interfaces: openViBE as a platform for a P300 speller," B.S. thesis ,medizinische informatik ,Heilbronn University of Applied Sciences,Heilbronn, 2012.

B. Graimann, B. Allison, and G. Pfurtscheller, "Brain - computer interfaces: a gentle introduction," Neurobionics Biomed. Eng. Neural Prostheses, pp. 231-236, 2010, doi: 10.1007/9783-642-02091-9 1 . 
[7] D. Zapała et al., "The effects of handedness on sensorimotor rhythm desynchronization and motor-imagery BCI control," Sci. Rep., vol. 10, no. 1, pp. 1-11, 2020, doi: 10.1038/s41598-02059222-w.

[8] D. Huang, K. Qian, O. Bai, D.-Y. Fei, W. Jia, and $\mathrm{X}$. Chen, "Electroencephalography (EEG)-based brain-computer interface (BCI): A 2-D virtual wheelchair control based on event-related desynchronization/synchronization and state control," IEEE Trans. Neural Syst. Rehabil. Eng., vol. 20, no. 3, pp. 379-388, 2012, doi: 10.1109/TNSRE.2012.2190299.

[9] J. R. Wolpaw and D. J. McFarland, "Control of a two-dimensional movement signal by a noninvasive brain-computer interface in humans," Natl. Acad Sci., vol. 101, no. 51, pp. 1784917854, 2004, doi: 10.1073/pnas.0403504101.

[10] D. J. McFarland, W. A. Sarnacki, and J. R. Wolpaw, "Electroencephalographic (EEG) control of three-dimensional movement," Neural Eng., vol. 7, no. 3, p. 036007, May 2010, doi: 10.1088/1741-2560/7/3/036007.

[11] A. S. Royer, A. J. Doud, M. L. Rose, and Bin He, "EEG Control of a Virtual Helicopter in 3Dimensional Space Using Intelligent Control Strategies," IEEE Trans. Neural Syst. Rehabil. Eng., vol. 18, no. 6, pp. 581-589, Dec. 2010, doi: 10.1109/TNSRE.2010.2077654.

[12] K. Lafleur, K. Cassady, A. Doud, K. Shades, E. Rogin, and B. He, "Quadcopter control in threedimensional space using a noninvasive motor imagery-based brain-computer interface," Neural Eng., vol. 10, no. 4, p. 15, Aug. 2013, doi: 10.1088/1741-2560/10/4/046003.

[13] A. J. Doud, J. P. Lucas, M. T. Pisansky, and B. $\mathrm{He}$, "Continuous three-dimensional control of a virtual helicopter using a motor imagery based Brain-Computer interface," in 2011 5th International IEEE/EMBS Conference on Neural Engineering, 2011, vol. 6, no. 10, pp. 364-367, doi: 10.1371/journal.pone.0026322.

[14] A. Akrami, S. Solhjoo, A. Motie-Nasrabadi, and M.-R. Hashemi-Golpayegani, "EEG-based mental task classification: Linear and nonlinear classification of movement imagery," in Annual International Conference of the IEEE Engineering in Medicine and Biology - Proceedings, 2005, vol. 7, pp. 4626-4629, doi: 10.1109/iembs.2005.1615501.

[15] K. P. Thomas, C. Guan, C. T. Lau, A. P. Vinod, and K. K. Ang, "A new discriminative common spatial pattern method for motor imagery braincomputer interfaces," IEEE Trans. Biomed. Eng., vol. 56, no. 11, pp. 2730-2733, 2009, doi: 10.1109/TBME.2009.2026181.

[16] M. Tangermann et al., "Review of the BCI competition IV," Front. Neurosci., vol. 6, pp. 131, doi: 10.3389/fnins.2012.00055.

[17] K. B. Böcker, J. A. van Avermaete, and M. M. van den Berg-Lenssen, "The international 10-20 system revisited: Cartesian and spherical co- ordinates," Brain Topogr., vol. 6, no. 3, pp. 231235, doi: 10.1007/BF01187714.

[18] C. Brunner, A. Delorme, and S. Makeig, "EEGLAB -an open source matlab toolbox for electrophysiological research," Biomed. Eng. / Biomed. Tech., vol. 58, pp. 12-13, 2013, doi: 10.1515/bmt-2013-4182.

[19] M. Kaur, S. S. Sidhu, and N. S. Ghumman, "Analysis of EEG signals using EEGLAB," Int. J. Adv. Res. Comput. Sci. Softw. Eng., vol. 5, no. 6, pp. 999-1002, 2015, [Online]. Available: www.ijarcsse.com.

[20] G. G'omez-Herrero, "Automatic Artifact Removal (AAR) toolbox v1.3 (Release 09.12.2007) for MATLAB," user guide,Tampere University of Technology.

[21] A. Delorme and S. Makeig, "EEGLAB: an open source toolbox for analysis of single-trial EEG dynamics including independent component analysis," J. Neurosci. Methods, vol. 134, no. 1, pp. 9-21, 2004, doi: 10.1016/j.jneumeth.2003.10.009.

[22] M. Bhardwaj and A. K. Nadir, "Analyzing electroencephalogram signal using EEG Lab," Sensors Transducers J. (ISSN 1726- 5479), vol. 100, no. 1, pp. 51-57.

[23] Y. Yang, "EEG signal analysis for brain-computer interfaces for large public applications," Signal Image Process. Télécom ParisTech, [Online]. Available: https://pastel.archives-ouvertes.fr/tel01234955.

[24] P. He, G. Wilson, and C. Russell, "Removal of ocular artifacts from electro-encephalogram by adaptive filtering," Med. Biol. Eng. Comput., vol. 42, no. 3, pp. 407-412, 2004, doi: 10.1007/BF02344717.

[25] B. Xu and A. Song, "Pattern recognition of motor imagery EEG using wavelet transform," $J$. Biomed. Sci. Eng., vol. 01, no. 01, pp. 64-67.May 2008, [Online]. Available: https://doi.org/10.4236/jbise.2008.11010.

[26] A.Sivakamil and S. S. Devi, "Analysis of EEG for motor imagery based classification of hand activities," Int. J. Biomed. Eng. Sci., vol. 2, no. 3, pp. 11-22, Jul .2015.

[27] S. Paszkiel, Analysis and classification of EEG signals for brain-computer interfaces, 1 st ed. Springer, Cham, 2020.

[28] E. R. Miranda, Guide to brain- computer music interfacing, 1st ed. London Heidelberg New York Dordrecht: Springer, London, 2014. 